\title{
Leserbrief
}

\section{Keine Hörgeräteversorgung ohne HNO-Arzt}

\author{
In der letzten Ausgabe der HNO-Nachrichten erläuterte Prof. Jürgen \\ Kieß-ling die Neufassung der Hilfsmittel-Richtlinie und die sich dar- \\ aus ergebenden Änderungen und Einschnitte. Zu diesem Beitrag er- \\ reichte uns zwischenzeitlich die folgende Zuschrift von Rechtsanwalt \\ Dr. A. Wienke. Anschließend nimmt Prof. Kießling dazu nochmals \\ Stellung.
}

In seinem Beitrag zur Neufassung der Hilfsmittel-Richtlinie befasst sich Herr Prof. Kießling u.a. mit den Neuregelungen der Hilfsmittel-Richtlinie. Er behauptet, dass nach den Neuregelungen eine HNO-ärztliche Verordnung nicht für Folgeverordnungen gelte, da das SGB V einen solchen ärztlichen Verordnungsvorbehalt für Hilfsmittel nicht vorsehe.

Diese Ausführungen sind sachlich falsch. Der Verordnungsvorbehalt gilt auch für Folge- und Wiederversorgungsfälle. Daher sei klarstellend auf Folgendes hingewiesen:

Nach § 27 Abs. 1 der ursprünglich beschlossenen Neufassung der Hilfsmittelrichtlinien in der Fassung vom 21.12. 2011 sollte die Abgabe von Hörhilfen zu Lasten der GKV stets eine vertragsärztliche Versorgung voraussetzen. Hierauf sollte nur verzichtet werden können bei Verlust oder irreparablem Defekt des bisher getragenen Gerätes, sofern die letzte Verordnung bei Kindern nicht länger als vier Jahre und bei Erwachsenen nicht länger als sechs Jahre zurückliegt. In den dazu geführten Beratungen hatten u.a. die Bundesärztekammer und die ärztlichen Sachverständigen darauf hingewiesen, dass die Bedeutung der ärztlichen Verordnung über die Feststellung einer Kausalität zwischen Hörbehinderung und Krankheit hinausgehe und die ärztliche Verordnung immer die Indikationsstellung für die konkret benötigte Hörhilfe sei, die ausschließlich Ärzten vorbehalten sei. Im zuständigen Ausschuss des Ge-meinsamen Bundesausschusses hat man sich dann auf die vorgenannte Formulierung des $\S 27$ Abs. 1 verständigt.

Diese Neufassung ist dann vom Bundesgesundheitsministerium kritisiert worden, weil die Ausführungen in den tragenden Gründen zu dieser Neufassung nicht konsistent waren, vielmehr den Schluss zuließen, dass eine vertragsärztliche Verordnung bei Folgeversorgungen generell nicht für notwendig angesehen werde.

Tatsächlich beinhalten die tragenden Gründe zum ursprünglichen Beschluss des Gemeinsamen Bundesausschusses vom 21.12.2011 eine Aneinanderreihung verschiedener Auffassungen zur Frage des Arztvorbehaltes bei Folge- und Wiederversorgungsfällen. Dabei wird u.a. auf eine angeblich bestehende Auffassung des Bundessozialgerichts zu $§ 15$ $S G B V$ verwiesen, wonach das SGB V einen ärztlichen Verordnungsvorbehalt bei der Hilfsmittelversorgung nicht vorsehe. Diese Interpretation ist schlicht falsch. Zu verweisen ist auf die Entscheidung des Bundessozialgerichts vom 16.09.1999. In diesem Urteil ging es um die Übernahme der Kosten für ein ärztlich für erforderlich gehaltenes Luftreinigungsgerät. Dazu hat das BSG ausgeführt, dass der Arztvorbehalt des § 15 Abs. 1 SGB

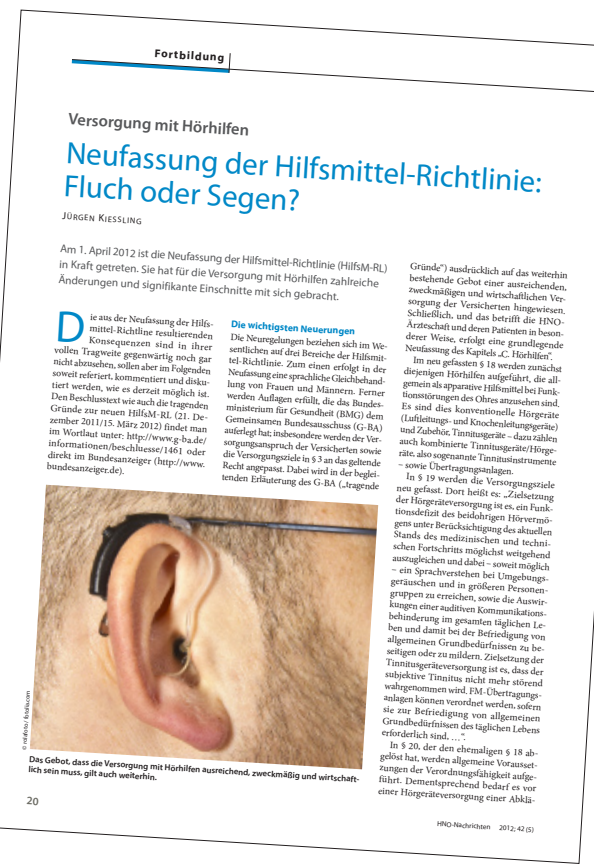

V nicht für "Hilfeleistungen anderer Personen" gemäß § 15 Abs. 3 SGB V für die Inanspruchnahme ", anderer Leistungen" gelte; es sagt aber nichts darüber aus, dass der Arztvorbehalt nicht für die Verordnung von Hörhilfen gelte. Die Bezugnahme auf die Rechtsprechung des BSG führt hier also nicht weiter. Vielmehr gilt der in $\S 15$ Abs. 1 SGB V generell und in $\S$ 73 Abs. 2 Nr. 7 SGB V speziell für Hilfsmittel geregelte ärztliche Verordnungsvorbehalt uneingeschränkt bei der Verordnung von Hörhilfen, auch in allen Folge- und Wiederversorgungsfällen.

Die von der Hörgeräteakustikerschaft in jüngster Zeit immer wieder aufgestellte Behauptung, dass eine ärztliche Verordnung in Folge- bzw. Wiederversorgungsfällen für Hörhilfen nicht erforderlich sei, ist damit ersichtlich falsch. Unzulässig ist daher natürlich auch der Verzicht auf die Abnahmeuntersuchung durch den HNO-Arzt. Soweit gesetzliche Krankenkassen in Verträgen mit Hörgeräteakustikern einen solchen Verzicht der HNO-ärztlichen Verordnung oder Kontrolluntersuchung propagieren, widerspricht dies eindeutig der Intention des Gemeinsamen Bundesausschusses und den Hilfsmittel-Richtlinien. HNO-Ärzte sollten daher stets darauf Wert legen, dassjeder Hörgeräteversorgung eine HNO-ärztliche Verordnung vorausgeht. Die Qualität der Hörgeräteversorgung und der Anspruch der Patienten auf eine optimale ärztliche Versorgung sollten es Wert sein.

\section{Rechtsanwalt Dr. A. Wienke}

Fachanwalt für Medizinrecht

Wienke \& Becker - Köln, Rechtsanwälte

Sachsenring 6

50677 Köln

Mail: AWienke@Kanzlei-WBK.de 